\title{
Reflets
}

Revue ontaroise d'intervention sociale et communautaire

\section{L'intégration des concepts autochtones dans le curriculum du travail social}

\section{Adje van de Sande et Gilles Renault}

Volume 4, numéro 1, printemps 1998

Intervention en contextes minoritaires

URI : https://id.erudit.org/iderudit/026203ar

DOI : https://doi.org/10.7202/026203ar

Aller au sommaire du numéro

Éditeur(s)

Reflets : Revue ontaroise d'intervention sociale et communautaire

ISSN

1203-4576 (imprimé)

1712-8498 (numérique)

Découvrir la revue

Citer cet article

van de Sande, A. \& Renault, G. (1998). L'intégration des concepts autochtones dans le curriculum du travail social. Reflets, 4(1), 164-173.

https://doi.org/10.7202/026203ar

Tous droits réservés (C) Reflets : Revue ontaroise d'intervention sociale et communautaire, 1998
Ce document est protégé par la loi sur le droit d'auteur. L'utilisation des services d'Érudit (y compris la reproduction) est assujettie à sa politique d'utilisation que vous pouvez consulter en ligne.

https://apropos.erudit.org/fr/usagers/politique-dutilisation/ 


\section{L'intégration des concepts autochtones dans le curriculum du travail social}

"La pratique du travail social auprès des peuples autochtones a subi plusieurs changements significatifs dans les dernières décennies. La profession est passée d'une tentative d'assimilation à un comportement d'accommodation et d'adaptation.»

\section{Adje van de Sande et Gilles Renault}

Professeurs, École de service social, Université Laurentienne

En Ontario, plus spécifiquement dans le Nord, les travailleurs sociaux sont appelés à travailler avec une population francophone et rurale, ce qui implique une certaine diversité culturelle. Cette population est composée, entre autres, de Québecois immigrés à cause de leur travail, de Scandinaves attirés par les emplois dans les mines et des peuples autochtones installés depuis toujours. Ces nations autochtones, principalement des Cris et des Ojibways, ont recours à l'occasion aux services d'agences pour nonautochtones. Les travailleurs sociaux ont alors la responsabilité de comprendre la culture, parfois la langue, mais surtout un mode d'intervention qui fait appel à la spiritualité.

La pratique du travail social auprès des peuples autochtones a subi plusieurs changements significatifs dans les dernières décennies. La profession est passée d'une tentative d'assimilation à un comportement d'accommodation et d'adaptation. Tout en étant très positifs, ces changements n'ont pas réussi à modifier l'enseignement du travail social. La profession ne reconnait pas encore la portée d'une formation ayant un modèle autochtone traditionnel de "guérison» dans son curriculum.

Le texte qui suit ne fait qu'expliquer le contexte de la spiritualité autochtone à travers la roue médicinale. La compréhension de cet encadrement à l'intervention ne signifie pas que l'on peut 
reproduire cette approche, mais bien que l'on peut s'en servir pour entreprendre un certain cheminement.

Les services sociaux pour autochtones ont jusqu'à récemment été livrés de façon paternaliste et avec une certaine indifférence au plan culturel. L'assimilation du peuple des Premières Nations dans la culture dominante était l'un des objectifs prédominants des interventions. La plupart des travailleuses sociales travaillant auprès des autochtones étaient de race blanche, de descendance européenne et sans grande connaissance des Premières nations. Tout en ayant les meilleures intentions du monde, ces travailleuses sociales croyaient vraiment que ce peuple s'en tirerait mieux s'il adoptait les valeurs et coutumes du peuple nord-américain.

En 1980, Philip Hepworth, oeuvrant pour le Conseil canadien du développement social, entreprit une importante recherche sur les soins nourriciers et l'adoption au Canada. Il démontra avec clarté la surreprésentation des enfants autochtones dans le système de d'aide sociale dans chacune des provinces (Hepworth 1980). Cette étude reste une des références les plus citées dans le domaine du bien-être à l'enfance au Canada.

Une autre étude entreprise par Patrick Johnston en 1983, intitulée Native Children and the Child Welfare System eut, elle aussi, un impact important sur la livraison du bien-être à l'enfance pour les enfants autochtones. L'auteur, en se servant des données recueillies lors du recensement canadien, parla même de «génocide culturel» en faisant allusion aux politiques visant les peuples

"On ne retrouve plus aujourd'hui cette pratique $d u$ retrait presque automatique de l'enfant de son milieu familial ou de sa réserve pour le placer avec une famille de race blanche loin de sa communauté.» autochtones (Johnston 1983). Cette recherche a souvent servi de référence lorsqu'on parle de contrôle de leur propre système de bien-être à l'enfance par le peuple autochtone.

On ne retrouve plus aujourd'hui cette pratique du retrait presque automatique de l'enfant de son milieu familial ou de sa réserve pour le placer avec une famille de race blanche loin de sa communauté. La plupart des provinces reconnaissent l'importance du respect de l'héritage de l'enfant. En Ontario, cette reconnaissance est même inscrite dans La loi sur les services aux enfants et aux familles de 1986. 
"À quelques exceptions près, les étudiantes en service social (ou travail social) apprennent surtout les modèles traditionnels d'intervention qui sont essentiellement ethnocentriques.»
Il est évident que le peuple dominant n'a pas respecté l'héritage culturel et les traditions des peuples autochtones. Par contre, les gouvernements et les travailleuses sociales soutiennent et encouragent aujourd'hui le développement de programmes de prévention qui sont culturellement appropriés. Ces services s'adressent spécifiquement à la maltraitance des enfants dans les communautés autochtones (van de Sande et al 1989).

Une grosse partie du problème est enracinée dans le programme de formation pour travailleuses sociales qui ignore les concepts aborigènes comme élément essentiel et légitime dans le développement du curriculum. À quelques exceptions près, les étudiantes en service social (ou travail social) apprennent surtout les modèles traditionnels d'intervention qui sont essentiellement ethnocentriques. Les diplômés de ces programmes travaillent à partir de ces modèles et ne tiennent pas compte des différences culturelles et des traditions des différents groupes ethniques, y compris les peuples des Premières nations.

\section{Les principes autochtones}

Avant d'entreprendre la description de la roue médicinale, il est important de bien comprendre la perspective autochtone quant à la vision que ces gens ont d'eux-mêmes et de leur relation avec l'environnement. Contrairement à la pensée occidentale, le peuple autochtone ne se considère pas comme une entité séparée de son environnement. Chez les autochtones, on ne retrouve aucune séparation entre le monde spirituel et le monde vivant.

Le concept d'esprit reste souvent particulièrement difficile à comprendre pour la travailleuse de race blanche. Selon la pensée autochtone, les esprits sont bel et bien vivants. La travailleuse sociale pourrait interpréter cette perspective comme étant une religion. Or, catégoriser la pensée traditionnelle autochtone comme étant «religieuse» démontre une incompréhension totale du monde aborigène. La spiritualité n'est pas seulement une façon de voir le monde mais bien une façon de le vivre. 


\section{La roue médicinale}

La roue médicinale est un ancien symbole utilisé par presque toutes les nations aborigènes de l'Amérique du Nord et de l'Amérique du Sud. On retrouve plusieurs versions de cette roue. Celle que nous présentons nous parvient d'une femme Anisnabe (Ojibway) de la réserve Sagamok First Nation, à quelques 100 kilomètres à l'ouest de Sudbury dans le Nord de l'Ontario, lors d'un cercle de guérison pour parents. Le livre de Bopp, Bopp, Brown et Lane, The Sacred Tree (1984), a aussi servi de guide pour décrire ce qui suit.

Figure 1 - La roue médicinale

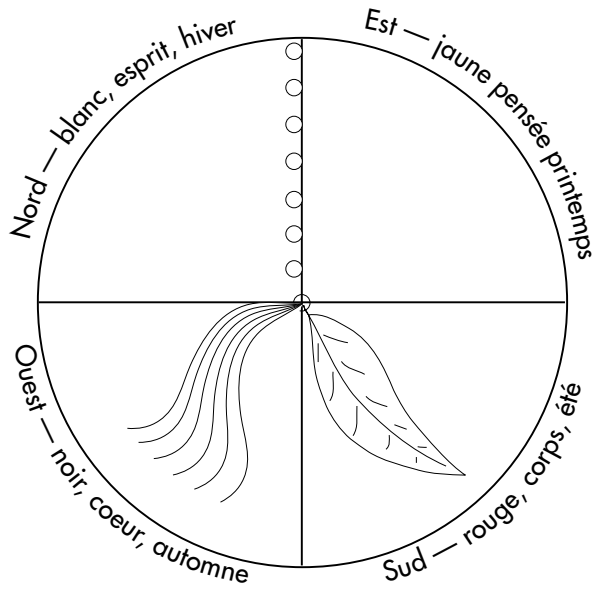

Le diagramme démontre clairement que la roue est essentiellement un cercle qui se divise en quatre parties égales avec des lignes qui s'entrecroisent. Le cercle signifie le chemin de la vie qui va toujours dans le sens des aiguilles d'une montre. Le bois du cercle est enveloppé de matériel de quatre différentes couleurs. Le quartier supérieur est blanc. Le blanc représente l'esprit, l'hiver et la direction nord. Le quartier à la droite de ce dernier est jaune et symbolise la pensée, le printemps et la direction est. Le quartier du bas est rouge et signifie le corps, l'été et la direction sud. 
Finalement, le dernier quartier est de couleur noire et fait allusion au coeur, à l'automne et à la direction ouest. Les quatre couleurs représentent également les quatre races du monde : blanche, jaune, rouge et noire. Sur la ficelle blanche partant du centre et montant vers le haut, on retrouve sept coquillages représentant les sept grands-pères : l'éternité, la sagesse et la connaissance, l'amour et la confiance, l'humilité et la patience, le courage et la bravoure et finalement le respect. Toutes ces doctrines ont leur opposé. Par exemple, l'opposé de l'amour serait la jalousie et la possessivité. Les sept coquillages symbolisent aussi les sept étapes de la vie, chacune ayant sept ans. Chaque étape a ses propres activités et ses propres défis à surmonter. Deux autres fils partent du centre, un bleu qui représente le ciel et un vert qui signifie la nature. Une plume est attachée au centre du cercle. Cette plume est le souffle du créateur. Ce souffle représente l'harmonie entre les gens et toute la création.

\section{Le chiffre quatre (4)}

"Comme on peut le voir par la construction de la roue, le chiffre quatre (4) s'y retrouve souvent. Ce symbole très puissant pour les peuples autochtones représente la façon selon laquelle notre monde est divisé.»
Tel que nous l'expliquent Bopp, Bopp, Brown et Lane (1984), les symboles nous apportent une compréhension du monde. Tous les peuples se servent de symboles et ils attribuent une certaine signification à leurs coutumes. Notre capacité de comprendre les peuples de ce monde serait sans doute très limitée sans l'aide de ces symboles.

Comme on peut le voir par la construction de la roue, le chiffre quatre (4) s'y retrouve souvent. Ce symbole très puissant pour les peuples autochtones représente la façon selon laquelle notre monde est divisé. Les quatre couleurs, tel que souligné auparavant, signifient les quatre races de la terre. Le cercle dans lequel se retrouvent ces quatre races symbolise l'appartenance à la même famille: "All are brothers and sisters living on the same Mother Earth" (Bopp, Bopp, Brown \& Lane 1984:10). Les quatre saisons sont aussi représentées par ces quatre couleurs: le blanc pour l'hiver lorsque la Nature s'endort et se regénère; le jaune 
pour le printemps, un temps d'activité intense avec le regain de vie de la Nature; le rouge qui signifie l'été avec la fertilité des terres; le noir qui symbolise l'automne avec les récoltes et l'hiver qui se prépare pour un autre long repos.

Selon les enseignements de la roue médicinale, notre univers est composé de quatre éléments: la terre, l'air, l'eau et le feu. Le Créateur nous les a donnés en cadeau et notre vie en dépend. La roue nous transmet aussi les quatre aspects de la nature humaine: physique, mental, spirituel et émotionnel. Toujours selon Bopp, Bopp, Brown et Lane (1984), ces quatre composantes, si elles sont développées de façon égale dans une vie bien équilibrée, font de l'individu un être de bonne volonté.

\section{Les quatre directions}

Le chiffre quatre représente également les quatre directions ou points cardinaux. La personne avance dans la vie comme elle marche autour de la roue. Chacune des directions est reliée à une certaine facette de la vie de l'individu et de son épanouissement personnel (Bopp, Bopp, Brown \& Lane 1984).

L'Est, les premiers pas de la vie, apporte une innocence, une spontanéité, une joie. On y retrouve une certaine naïveté, une acceptation inconditionnelle et une vulnérabilité. C'est l'enfance, malheureusement trop courte.

Après un certain apprentissage dans cette direction, nous changeons de direction et nous nous dirigeons vers le Sud. Maintenant devenu jeune adulte, l'espoir et la détermination sont à l'honneur. Cette étape de vie devient l'une des plus productives, avec tous ces buts que l'on se fixe. Nous avons appris à comprendre et exprimer nos émotions ainsi qu'à être sensible envers celles des autres.

Encore une fois et souvent de façon trop rapide, nous devons nous diriger vers une autre direction, l'Ouest, qui symbolise la noirceur et l'inconnu. Nous questionnons maintenant nos choix, 
«En écoutant ces leçons de la vie, nous apprendrons à vivre de façon plus équilibrée et à répondre à nos besoins ainsi qu'à ceux des autres.» le sentier que nous nous sommes donné. Durant cette période de notre vie, nous avons besoin d'être seul, de réfléchir sur nos gestes ainsi que sur les moyens à prendre pour rétablir nos priorités. Nous apprécions mieux notre spiritualité; nous nous dotons d'un code moral personnel et reconnaissons les valeurs universelles de la vie. Un respect pour l'enseignement des ainées et des aînés commence à se développer.

Finalement, arrive la direction du Nord. Nous sommes maintenant un aîné avec toute la sagesse qui vient avec l'âge. Nous comprenons mieux la chimie de la vie, comment la vivre de façon équilibrée et en harmonie avec la nature. Nous devenons l'enseignant qui partage ses connaissances avec ceux et celles qui l'entourent.

C'est ce que la roue représente. En écoutant ces leçons de la vie, nous apprendrons à vivre de façon plus équilibrée et à répondre à nos besoins ainsi qu'à ceux des autres. La roue médicinale, c'est l'univers, c'est soi-même: l'esprit, la pensée, le corps et le coeur.

\section{L'application de la roue}

Le récit qui suit viendra illustrer l'utilisation de la roue médicinale en tant qu'outil de counselling. L'exemple sera décrit très brièvement et de façon plutôt simpliste. Nous espérons que vous pourrez quand même apprécier le potentiel d'un tel encadrement en travail social. Il faut aussi spécifier qu'afin de pouvoir intervenir dans un tel cadre, il faut avoir reçu une formation spirituelle sous la direction des aînés. Le programme «Native Human Services» de l'École de service social de l'Université Laurentienne offre la possibilité d'obtenir une telle formation ou expérience.

Anna, âgée de dix-huit ans, mère d'un enfant de deux ans, vit sans conjoint. La communauté autochtone qui les héberge est constituée de quelque 600 habitants. On retrouve souvent Anna sans son fils, avec ses amis, et elle rentre à la maison très 
tard en soirée. Elle prend pour acquis que les voisins s'occuperont de son fils.

Un des principes traditionnels du peuple autochtone est que l'enfant est la responsabilité de toute la communauté. Ce principe est bel et bien accepté dans la communauté d'Anna et cela ne cause aucun problème. Il $\mathrm{y}$ a toutefois des occasions où les voisins ne sont pas disponibles et le fils d'Anna est laissé sans surveillance pendant plusieurs heures. Les voisins de la jeune femme lui en ont parlé mais sont restés sans réponse de sa part. N'ayant plus le choix, les voisins d'Anna se sont plaints au Conseil de bande afin que celui-ci fasse intervenir la travailleuse sociale de la communauté. Celle-ci réussit à convaincre Anna de participer à un Cercle de parents. Ce cercle, tel qu'on l'a expliqué à Anna, est constitué de plusieurs jeunes mères qui ont comme but d'apprendre les habiletés parentales traditionnelles autochtones. Anna connaissait très bien l'aînée qui menait le cercle pour l'avoir vue à plusieurs activités communautaires. Elle s'est aperçue tout de suite que l'on portait beaucoup de respect envers cette femme. Pendant la première session, comme c'était la coutume, l'aînée a commençé par donner le récit de sa propre histoire, notamment sa négligence envers ses propres enfants à cause d'un problème d'abus d'alcool. Elle avait éprouvé des problèmes si graves que la Société d'aide à l'enfance avait dû lui retirer ses enfants jusqu'à ce qu'elle ait réglé ses problèmes de consommation. Quelques années plus tard, elle avait participé à un cercle tel que celui-ci et avait été initiée à la roue médicinale. Cette expérience avait changé toute sa vie. Elle alla jusqu'à dire que si elle avait connu les enseignements de la roue médicinale avant, elle n'aurait probablement jamais perdu la garde de ses enfants. C'est à ce momentlà qu'elle avait décidé d'apprendre tout ce qu'elle pouvait au sujet de la roue médicinale et de partager ses connaissances avec d'autres dans le besoin.

À la suite de son récit, les participantes purent partager leurs inquiétudes et leurs attentes au sein du cercle. Les membres ont vite compris que l'aînée n'était pas là pour les juger mais simplement pour leur permettre d'éviter ses erreurs. L'aînée montra ensuite une roue médicinale qu'elle avait faite, il y a 
plusieurs années. Elle résuma chacune des parties de la roue : les couleurs, les quatre directions et les sept coquillages. Selon les enseignements de la roue, la plume au centre représentait le souffle du Créateur, celui qui donne la vie aux enfants. Elle leur expliqua aussi que les enfants ne sont pas la propriété des parents mais bien un prêt du Créateur. L'aînée continua en leur disant que c'était le rôle des parents de voir au bien-être des enfants, de bien les préparer à la vie, de les amener à participer et à contribuer à la communauté afin de garder cette communauté forte. Selon elle, le Créateur a le choix de ramener les enfants parmi les esprits si ceux-ci sont négligés.

$\mathrm{Au}$ fur et à mesure que la discussion continuait, certaines participantes exprimèrent leurs inquiétudes quant au réalisme de ces principes dans une vie tellement plus complexe. Cela devenait de plus en plus difficile d'élever un enfant sans conjoint. L'aînée leur donna raison mais leur fit remarquer qu'elles n'étaient pas seules. Plusieurs membres furent d'accord et décidèrent de se former un groupe de soutien afin de s'entraider. De cette façon, chacune des participantes pouvait contribuer en gardant le groupe d'enfants pendant que les autres mères vaquaient à d'autres occupations.

Même si le cercle continua quelques temps,Anna avait compris l'importance de ne pas négliger son fils. Toute la communauté souffrirait en même temps que son fils. Elle pouvait maintenant compter sur des amies pour l'aider avec son fils.

\section{Conclusion}

La roue médicinale est maintenant utilisée dans plusieurs centres de traitement à travers les communautés autochtones. On y retrouve très peu de modèles théoriques et ces méthodes restent aussi très difficiles à évaluer de façon empirique. Il reste néanmoins que cette approche a un impact considérable dans les communautés autochtones. 
Pour ceux de nous qui pensent que nos interventions doivent être basées sur des méthodes scientifiques, le concept d'interdépendance avec l'environnement, l'importance des valeurs et des principes de base et le besoin de bâtir une communauté plus forte à travers la contribution de tous sont de riches leçons qui ont leur place dans notre répertoire. Si ces principes devenaient la base de l'enseignement en travail social, notre profession et notre clientèle s'en porteraient mieux.

\section{Bibliographie}

BOPP, J., M. BOPP, L. BROWN et P. LANE (1984). The Sacred Tree, Lethbridge, Alberta: Four Winds Development Project.

HEPWORTH, P. (1980). Foster Care and Adoption in Canada, Ottawa, The Canadian Council on Social Development.

JOHNSTON, P. (1983). Native Children and the Child Welfare System,Toronto, The Canadian Council on Social Development, James Lorimer \& Company, Publishers.

MINISTÈRE DES SERVICES SOCIAUX ET COMMUNAUTAIRE DE L'ONTARIO (1986).

Résumé de la loi sur les services à la famille et aux enfants, Toronto, Gouvernement de l'Ontario.

VAN DE SANDE,A., J. NAIDOO et F. GLOADE (1989). «The Adoption of Aboriginal Children: An Annotated and Selected Bibliography", Native Studies Review, vol. 5, no 2,163-183. 